Abstract

\title{
Nutritional Symbionts Confer Structural Defence Against Predation and Fungal Infection in a Grain Pest Beetle ${ }^{+}$
}

\author{
Sthandiwe Nomthandazo Kanyile 1, Tobias Engl 1,2 and Martin Kaltenpoth 1,2,* \\ ${ }^{1}$ Evolutionary Ecology, Institute of Organismic and Molecular Evolution, Johannes Gutenberg-University, \\ Mainz, Germany \\ 2 Department of Insect Symbiosis, Max-Planck-Institute for Chemical Ecology, Jena, Germany \\ * Correspondence: skanyile@uni-mainz.de \\ + Presented at the 1st International Electronic Conference on Entomology (IECE 2021), 1-15 July 2021; \\ Available online: https://iece.sciforum.net/.
}

Citation: Kanyile, S.N.; Engl, T.;

Kaltenpoth, M. Nutritional

Symbionts Confer Structural

Defence Against Predation and

Fungal Infection in a Grain Pest

Beetle, in Proceedings of the 1st In-

ternational Electronic Conference on

Entomology, 1-15 July 2021, MDPI:

Basel, Switzerland,

doi:10.3390/IECE-10525

Published: 1 July 2021

Publisher's Note: MDPI stays neutral with regard to jurisdictional claims in published maps and institutional affiliations.

Copyright: (C) 2021 by the authors. Submitted for possible open access publication under the terms and conditions of the Creative Commons Attribution (CC BY) license (http://creativecommons.org/licenses /by/4.0/).
Many insects benefit from bacterial symbionts that provide essential nutrients and thereby extend the hosts' adaptive potential and their ability to cope with challenging environments. However, the implications of nutritional symbioses for the hosts' defence against natural enemies remain largely unstudied. Here, we investigated if the cuticleenhancing nutritional symbiosis of the saw-toothed grain beetle Oryzaephilus surinamensis confers protection against predation and fungal infection. For this, we exposed agedefined symbiotic and symbiont-depleted (aposymbiotic) beetles to two antagonists that must actively penetrate the cuticle for a successful attack: wolf spiders (Lycosidae) and the fungal entomopathogen Beauveria bassiana. While young beetles suffered from high predation and fungal infection rates regardless of symbiont presence, symbiotic beetles were able to escape this period of vulnerability and reach high survival probabilities significantly faster than aposymbiotic beetles. To understand the mechanistic basis underlying these differences, we conducted a time-series analysis of cuticle development in symbiotic and aposymbiotic beetles by measuring cuticular melanisation and thickness. The results reveal that the symbionts accelerate their host's cuticle formation and thereby enable it to quickly reach a cuticle quality threshold that confers structural protection against predation and fungal infection. Considering the widespread occurrence of cuticle enhancement via symbiont-mediated tyrosine supplementation in beetles and other insects, our findings demonstrate how nutritional symbioses can have important ecological implications reaching beyond the immediate nutrient provisioning benefits.

Keywords: Oryzaephilus surinamensis; sawtoothed grain beetle; Bacteroidetes; Candidatus Shikimatogenerans silvanidophilus; symbiosis; mutualism; cuticle; structural defense 